\title{
The possible role of embryonic polarity axes for the normalization of tissue function induced by the interaction between human bilateral parts
}

\author{
Ming Cheh $\mathrm{Ou}^{1,2 *}$, Dennis $\mathrm{Ou}^{3}$ and Chung Chu Pang ${ }^{4}$ \\ ${ }^{1}$ Department of Obstetrics and Gynecology, Taipei City Hospital, Taiwan \\ ${ }^{2}$ Department of Obstetrics and Gynecology, Taipei Medical University, Taiwan \\ ${ }^{3}$ Department of Mechanical Engineering and Biomedical Engineering, Carnegie Mellon University, USA \\ ${ }^{4}$ Department of Obstetrics and Gynecology, Su Women Hospital, Taiwan
}

\begin{abstract}
Ou MC decrescendo phenomenon (OuDP) is produced by placing the contralateral hand over a diseased location (Ou MC handing remedy [Ou HR]) to produce a zone under the hand with decreased pain or inflammation. Our objective is to report a series of patients with various diseases that resolved with application of the Ou HR, and review other studies of the OuDP. During 2015, 18 patients with various diseases were treated at our clinic: 3 patients had infectious processes; 4 had joint and/or soft tissue pain; 3 had dysmenorrhea; 1 had preterm labor; 2 had abdominal pain/fullness after surgery; 1 had diabetes mellitus and difficulty walking; 2 had suspected malignancies and 1 had a malignancy. All patients were treated with the Ou HR. Of the 18 patients, 17 self-administered the HR and 1 had the Ou HR administered by a therapist. In all 18 patients the symptoms or disease process was improved or cured. Prior published studies including 121 patients indicated that the $\mathrm{Ou} \mathrm{HR}$ was effective in 92.3 to $100 \%$ of patients. Including prior studies, there were 3 patients with pathology-identified cancer: (1) uterine endometrioid carcinoma, stage IIIB, (2) uterine endometrial carcinoma, stage IA, (3) uterine leiomyosarcoma stage IB, All these patients showed suppression of the tumor with Ou HR treatment. The OuDP shows to accelerate the recovery of a number of disease states, and the effect mainly occurs with the use of the contralateral hand, which suggests that the axes of embryonic polarity may impart the OuDP leading to the normalization of tissue function. The normalization of the tumor cells and microenvironment function may make tumor cells conform to the regulations with apoptosis, metastasis suppression, preventing uninhibited proliferation, minimizing angiogenesis and supervision by host immunological systems, which suppresses the cancer. These findings warrant further investigation.
\end{abstract}

\section{Introduction}

The development patterning along the axes of embryonic polarity is essential for producing a normal anatomical structure and function. In a study of snapping shrimp (Alpheus heterochelis), the contralateral snapper claw was found to affect the development of a new claw after it was lost to achieve bilateral symmetry in both shape and fiber composition of the new claw [1]. Exposure to conspecific mutual interactions with other snapping shrimp also triggered such claw transformation. A study showed that itching of the hand in humans can be controlled by stimulation of the contralateral hand [2]. Other studies have shown that the mutual interaction of bilateral topographically symmetrical features of mammals may also induce and reinforce inflammatory reactions and pain sensations on the contralateral side of the body. Examples of such a phenomenon are contralateral arthritis induced by unilateral arthritis in rats, and symmetrical development of arthritis, pulmonary fibrosis, glomerulonephritis, and sympathetic ophthalmia in humans [3]. The mechanisms involved in the mutual interaction between bilateral parts of topographically symmetrical living beings remain largely unknown, though there are indications that such interaction may occur through neurological transmission [3].

In our prior studies, we have described the Ou MC decrescendo phenomenon (OuDP) and the Ou MC handing remedy (Ou HR) [4-7]. The HR involves placing the contralateral hand over a diseased body part. Placement of the hand initiates the decrescendo phenomenon, which leads to normalization of tissue function and resolution of the disease process. The Ou HR has been shown to improve or cure disease processes ranging from infection, to soft tissue and joint disease, to malignancy. Importantly, our prior studies have shown that the HR is inefficatious at producing the decrescendo phenomenon when the ipsilateral hand is used [4-7].

This effect is similar to the interactions between the two claws of snapping shrimps in which symmetry of both tissue composition and shape of the new claw with the contralateral claw is achieved [1].

The purpose of this report is to present a case series of patients with diverse disease processes who received resolution or improvement after being treated with the Ou HR. The report also reviews our prior studies showing that the effect mainly occurs when the contralateral hand is used, and discusses the potential mechanisms for this observation.

Correspondence to: Ou, Ming Cheh, Department of Obstetrics and Gynecology, Taipei City Hospital, Taipei City, Taiwan; E-mail: mcou@ym.edu.tw

Key words: infection, tissue function normalization, human bilateral parts interaction, ou mc decrescendo phenomenon, embryonic polarity axes

Received: December 01, 2015; Accepted: December 17, 2015; Published: December 21, 2015 


\section{Methods and patients}

In this report we present a case series of patients treated with the Ou HR at our institution in the year 2015. Although complementary therapy is exempt from informed consent by the law in Taiwan (Department of Health, Executive Yuan, Taiwan ROC, 1993) [8], all patients provided consent for treatment with the HR and to have the details of their cases, including radiographic images published.

Patients presented with diverse conditions including infections, joint and soft tissue pain, dysmenorrhea, preterm labor, suspected malignancy and malignancy. The Ou HR can be performed by the patient or a therapist. The technique is performed by placing the contralateral hand over the site of the lesion, typically 2-3 times a day. An illustration of application of the HR is shown in Figure 1, and practical points for HR application are summarized in Table 1. Ou MC manipulation [4] was also performed for patients with acute abdomen to demonstrate the effectiveness of ipsilateral and contralateal hand to induce OuDP.

\section{Case series}

\section{Infections}

A 54 year-old-male was seen with a nasopharyngeal infection that was unresponsive to 1 week of antibiotic therapy. After discontinuation of antibiotics, he performed the HR bilaterally, with the contralateral hands, for 1 minute on the facial maxilla and anterior neck 3 times a day. His symptoms resolved after 3 days of treatment, and did not recur.

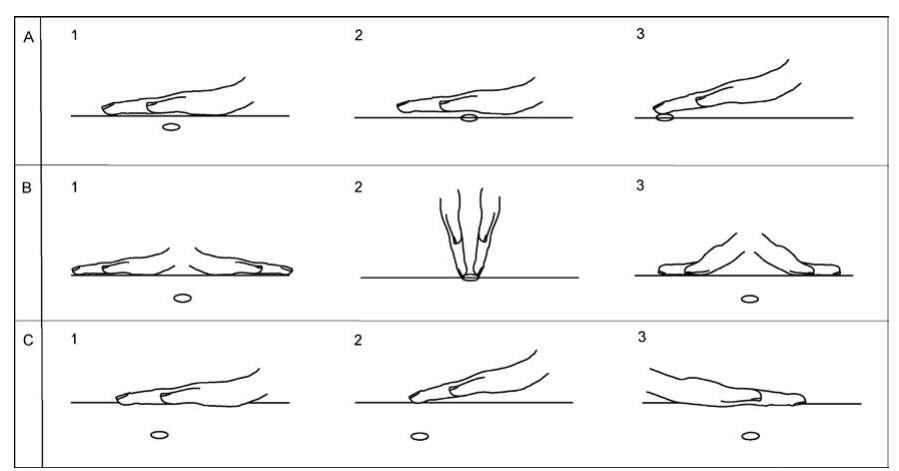

Figure 1. Illustration of the $\mathrm{Ou} M C$ handing remedy (Ou HR) technique. The $\mathrm{Ou} \mathrm{HR}$ is performed by placing the contralateral hand on the affected area (A1-A3). For lesions on or adjoining the midline of the body, the Ou HR is applied to one side of the lesion then to the other (B1-B3). The amount of pressure applied is commensurate with the depth of the lesion (C1-C3). Multiple fingers or the back of hand can be used if the palm cannot reach the affected area (A3-C3). For skin lesions, the palm may be kept about $0.5 \mathrm{~cm}$ over the lesion without touching the skin (A2). Small ellipse represents the lesion.
A 61-year-old male had a painful black necrotic cutaneous lesion measuring about $0.3 \times 0.4 \mathrm{~cm}$ on the left great toe. He performed the $\mathrm{HR}$ on the lesion with the contralateral hand for 2 minutes, twice a day. The lesion resolved after 4 days without the need for antibiotics.

A 63-year-old female had a 2 year history of relapsing oral cavity pain close to the bilateral intermaxillary commissure area. The pain did not resolve with antibiotics and analgesics. She discontinued antibiotics and performed the HR outside the oral cavity with the contralateral hand for 1 week. The pain persisted, and she then performed HR inside the mouth directly on the location of pain, and the pain completely remitted within 1 week.

\section{Joint and soft tissue pain}

A 57-year-old female had bilateral shoulder pain and lower back pain for 5 years. She performed the HR on each location, with the contralateral hand, for 1 minute, twice a day. The right shoulder pain and lower back pain resolved completely after 1 month, while the left shoulder pain persisted due to her right hand not being able to reach her left shoulder to perform the HR. Initially, this patient had erroneously performed the HR with the ipsilateral hand for 1 month, and complained of no pain alleviation for both shoulders.

An 86-year-old female was seen with bilateral knee joint pain for over 10 years. She performed the HR for 2-3 minutes, twice a day, with the contralateral hands on the knees. The bilateral joint pain was almost completely relieved after 1 week.

A 62 -year-old male had bilateral hip joint pain for about 8 months. The pain resulted in difficulty climbing stairs. He performed the HR for 1 minute, twice a day, with the contralateral hands on bilateral hip joints. The bilateral joint pain and climbing disability was almost completely relieved after 1 week. However, the joint pain of this patient occasionally could be relieved with the ipsilateral hand while recurred.

An 89-year-old male had a longstanding history of lower back pain. He was treated with the HR by a therapist using contralateral hands for 2-3 minutes, twice a day, and the pain was partially relieved after 1 week of treatment.

\section{Dysmenorrhea}

Three females, 34, 36, and 42 years of age, received endoscopic surgery for endometriosis and endometrial cysts and another 19-yearold female had dysmenorrheal for 2 years. All 3 patients that received surgery had a recurrence of dysmenorrhea after the surgery. All 4 of the patients performed the $\mathrm{HR}$ for 2-3 minutes, twice daily, on the bilateral lower abdomen using contralateral hands. All 4 patients experienced a resolution of their dysmenorrhea.

\section{Preterm labor}

A 36-year-old female with a breech pregnancy at 32 weeks' gestation

Table 1. Practical points for the self-administered application of the Ou MC handing remedy (OuHR)

1. The HR is performed by the patients themselves or the therapist [4] by placing the contralateral hand directly on the affected area. Placement of the hand initiates the Ou MC decrescendo phenomenon.

2. For lesions on or adjoining the midline of the body, the Ou HR is applied first to one side of the lesion and then the other, using the contralateral hands.

3. The effects of the Ou HR are related to the duration and frequency of administration, and the distance between the hand and the lesion.

4. If Ou HR is not efficacious, measures such as getting the hand nearer to the lesion, and increasing the duration or frequency of administration may be helpful.

5. Different positioning may be useful when performing the HR. For example, when treating lumbosacral pain, the patient may wish to lie down supine with the contralateral hand beneath the affected lumbosacral and adjoining areas.

6. Severe emotional disturbance may possibly affect effectiveness. 
was seen with preterm labor. The uterine contractions were poorly controlled with tocolytic agents (ritodrine and nifedipine) and the cervical length decreased from $4.0 \mathrm{~cm}$ to $3.2 \mathrm{~cm}$. She performed the HR 5 minutes on each side of the uterus, 3 times a day, using contralateral hands. The frequency of the uterine contraction decreased, and the ritodrine and nifedipine requirements were reduced. The pregnancy was maintained to 36 weeks, and an infant was delivered by Cesarean section with Apgar scores of 9 and 10 at 1 and 5 minutes, respectively. Of note, the patient initially performed the HR with the ipsilateral hands and uterine contractions increased in frequency during this time.

\section{Abdominal fullness or pain after surgery}

A 44-year-old female experienced frequent abdominal fullness after a right oophorectomy and lymph node dissection for right ovarian cancer stage IA in 2008. She performed the HR for 2 to 3 minutes, 2 to 3 times per day on the bilateral and lower abdomen. The abdominal fullness was relieved with obvious resolution of her abdominal distension.

A 55-year-old female patient experienced frequent lower pelvic pain after subtotal hysterectomy for uterine myoma. She performed the HR for 2 to 3 minutes, 2 to 3 times per day on the lower abdomen and the pelvic pain subsided without antibiotics or analgesics.

\section{Diabetes mellitus and walking disability}

A 76-year-old female with a 30 year history of diabetes mellitus complained of difficulty walking with swelling and color changes of both feet for 6 months. She performed the HR for both feet, legs, thighs, lower abdomen, and bilateral costovertebral area for 2 minutes on each site 2 to 3 times per day and was able to walk with less difficulty after 1 week.

\section{Suspected malignancy}

A 49-year-old menopausal female was found to have a right ovarian tumor (radiodensity $=70$ Hounsfield units with computed tomography scan) on vaginal sonography that measured $4.7 \times 4.0 \times 4.3 \mathrm{~cm}$ (Figure 2). She performed the HR with the contralateral hand on the lesion side of her lower abdomen for 5 minutes, twice a day. Vaginal sonography after 1 week revealed the lesion had decreased in size to $3.4 \times 3.9 \times 3.6 \mathrm{~cm}$.
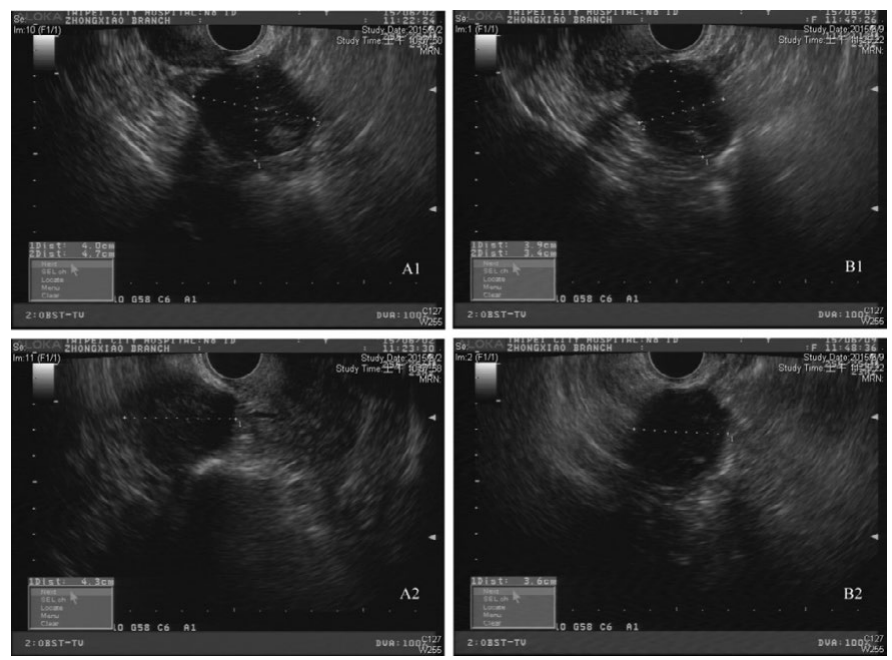

Figure 2. A 49-year-old menopausal female was found to have a right ovarian tumor on vaginal sonography that measured $4.7 \times 4.0 \times 4.3 \mathrm{~cm}$ (A1, A2). After performing the $\mathrm{Ou}$ $\mathrm{MC}$ hand remedy for 5 minutes, twice daily, for 1 week the lesion had decreased in size to $3.4 \times 3.9 \times 3.6 \mathrm{~cm}(\mathrm{~B} 1, \mathrm{~B} 2)$. Tumor radio density=70 Hounsfield units.
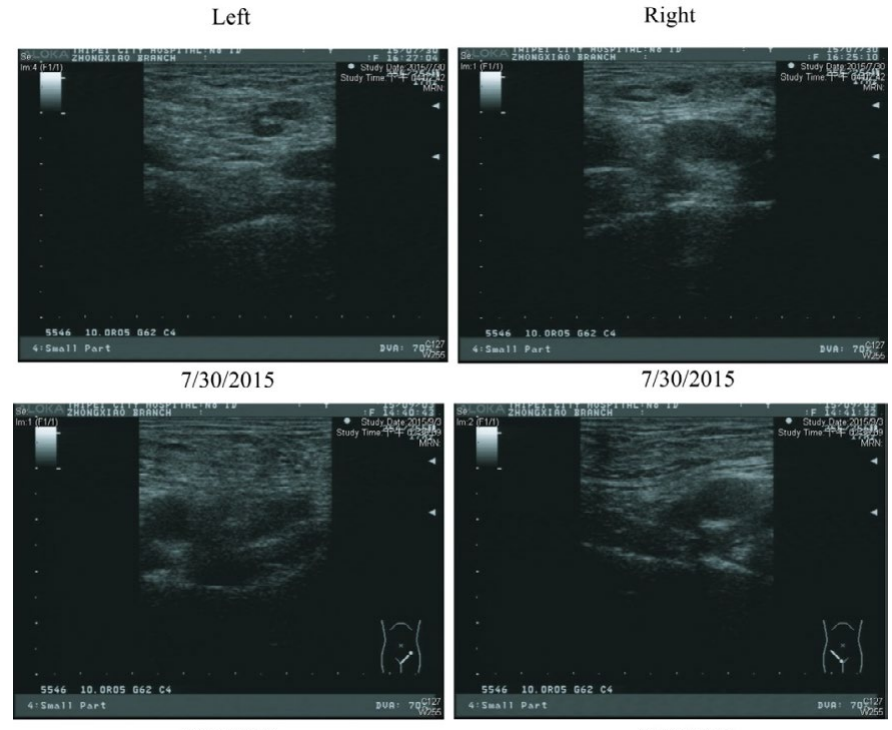

9/03/2015

Figure 3. A 52-year-old female post radical hysterectomy and bilateral pelvic lymphadenectomy for stage IB cervical cancer 9 years prior presented with painless enlarged immovable lymph nodes in the bilateral inguinal areas. Sonography on 7/30/2015 showed bilateral enlarged inguinal lymph nodes with evidence of tumor metastasis (upper panel). She performed the Ou MC hand remedy 2-3 minutes, twice daily, with contralateral hands, on the areas of the enlarge lymph nodes. Sonography after 4 weeks showed no enlarged lymph nodes in the bilateral inguinal areas (lower panels).

A 52-year-old female had a radical hysterectomy and bilateral pelvic lymphadenectomy 9 years prior for stage IB cervical cancer. She presented to our clinic with painless enlarged immovable lymph nodes in the bilateral inguinal areas. Sonography showed bilateral enlarged inguinal lymph nodes with evidence of tumor metastasis. She performed the HR 2-3 minutes, twice daily, with contralateral hands, on the areas of the enlarge lymph nodes. The size of lymph nodes decreased, and sonography after 4 weeks showed no enlarged lymph nodes in the bilateral inguinal areas (Figure 3).Though inguinal lymph node cancer metastasis was suspected, lymph node inflammatory disease could not be excluded; however, the patient refused further therapy, including a biopsy.

\section{Malignancy}

A 53-year-old female experienced vaginal bleeding for 2 months, and endometrial curettage revealed well-differentiated endometrial carcinoma. She administered the HR on the lower abdomen over the area of the uterus with contralateral hands for 5 minutes, twice daily, and the uterine bleeding stopped in 1 week and did not recur for 1 month before moving to other hospital.

A summary of the results of the 18 patients in the case series is shown in Table 2 .

\section{Acute abdomen diagnosed with $\mathrm{Ou}$ MC manipulation}

Another 8 female patients with pelvic inflammatory diseases and abdominal pain, 43.4 years old (SD, 21.2 years) in average, were seen in 2015. These patients received Ou MC manipulation to separate the pelvic organs from other abdominal areas (Figure 4) and the separation with the contralateral hand produced the OuDP while the ipsilateral hand did not $[4,5]$. 
Table 2. Summary of 18 patients treated with the Ou MC handing remedy in 2015.

\begin{tabular}{|c|c|c|c|}
\hline \multirow{2}{*}{ Diseases } & \multicolumn{2}{|c|}{ Hand remedy administration } & Improved or cured \\
\cline { 2 - 4 } & Self & Therapist & 3 \\
\hline Infection & 3 & 0 & 4 \\
\hline Joint and soft tissue pain & 3 & 1 & 4 \\
\hline Dysmenorrhea & 4 & 0 & 1 \\
\hline Preterm labor & 1 & 0 & 2 \\
\hline Suspected malignancy & 2 & 0 & 1 \\
\hline Malignancy & 1 & 0 & 2 \\
\hline Abdominal pain/fullness & 2 & & 1 \\
\hline Diabetes/walking difficulty & 1 & & 18 \\
\hline Total & 17 & 1 & \\
\hline
\end{tabular}

\section{Ou MC decrescendo phenomenom}

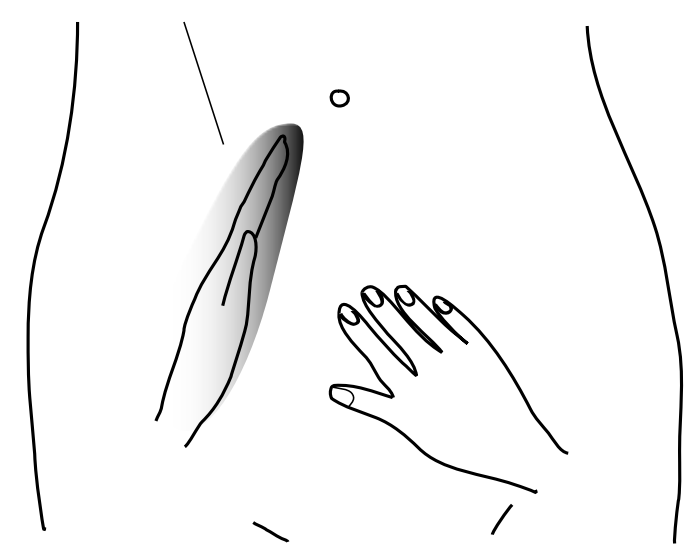

Figure 4. The Ou MC decrescendo phenomenon (OuDP) involves placing the contralateral hand of the examiner on the acute abdomen of female patients (Ou MC manipulation), which results in an alleviation of pain under the examining hand.

\section{Discussion}

In this report we have illustrated the effectiveness of the Ou HR for treating a variety of disease states. The HR induces the OuDP that we believe normalizes tissue function leading to healing. Importantly, the $\mathrm{HR}$ is effective when the contralateral hand is used-most cases using the ipsilateral hand produces no prominent effect. This is similar to the symmetrical effect seen in a species of snapping shrimp [1].

In a pilot study we showed the value of abdominal palpation with $\mathrm{Ou} \mathrm{MC}$ manipulation to help localize the tenderness associated with organs in the pelvis by isolating the visceral peritoneum and organs in women with pelvic inflammatory disease with an acute abdomen [4,5]. From January 2006 through December 2009, 113 women with an acute abdomen seen in the emergency department received traditional abdominal palpation and abdominal palpation with $\mathrm{Ou}$ MC manipulation [4,5]. Excluding cases with concurrent pelvic and nonpelvic organ disease, the sensitivity of abdominal palpation with $\mathrm{Ou}$ MC manipulation for the diagnosis of pelvic organ disease was significantly greater than that of traditional abdominal palpation $(P$ $=.003$ ). For 3 of 42 patients with both pelvic and nonpelvic pain (of the 113 patients), when the ipsilateral hand was used the origin of the pain could not be localized-only when the contralateral hand was used could the location of the pain be identified [4,5]. A subsequently study in which separate groups of 39 [6] and 40 [6,7] patients, respectively, with infectious and noninfectious processes showed that the Ou HR resolved symptoms in $92.3 \%$ and $100 \%$ of patients, respectively. Including prior studies [4-7], totally 56 patients receiving Ou MC manipulation or HR with contralateral as well as ipsilateral hand, application with contralateral hand resulted in OuDP for all the patients, while the ipsilateral hand resulted in OuDP for only one patient $(1 / 56,2.0 \%)$-Difference between contralateral and ipsilateral hand to induce OuDP, $P<0.001$, Paired t test (Table 3 ).

The Ou HR has also been shown to be effective in patients with malignancies. From 2011 to 2014, 4 females were instructed in the self-application of the HR for diseases including 1) stage IIIB uterine endometrioid carcinoma, 2) an isodense pancreatic nodule suspicious for stage IA pancreatic cancer, elevated CA199, and dilated tortuous main pancreatic duct, 3) stage IB uterine leiomyosarcoma with profuse bleeding, and 4) a suspicious gluteal metastatic lesion of chronic myelogenous leukemia [7]. The patients self-administered the HR, and in these patients: 1) The uterine endometrioid carcinoma regressed from stage IIIB to IA with 5 months treatment (Figure 5 and 6); 2) The pancreatic isodense lesion decreased in size from $1.6 \times 1.7$ $\mathrm{cm}$ to $1.0 \times 1.0 \mathrm{~cm}$, the CA199 level decreased from 1090 to $136 \mathrm{U} /$ $\mathrm{mL}$, and the main pancreatic duct diameter decreased from 0.39 to $0.14 \mathrm{~cm}$ with resolution of the tortuosity after 4 months of treatment (Figure 7); 3) The profuse bleeding due to the uterine leiomyosarcoma decreased immediately with application of the HR; and 4) The gluteal macular lesion in the patient with chronic myelogenous leukemia was eliminated with 2 weeks of treatment.

Alleviation of pain, cessation of uterine bleeding, improvement of dysfunction, and elimination of malignant changes using the $\mathrm{Ou}$ HR to produce the OuDP suggests the restoration of normal tissue function, which improved or cured various diseases in our studies. The restoration of normal tissue function may involve re-establishing host defense systems, which contribute to defense against microorganisms, inflammation, degenerative changes, and tumors [9]. The restoration of normal tissue function provided by the HR may require repeated session over a long period of time to produce a long-term effect.

The Ou HR appears to be consistently effective for treating a wide variety of diseases, and the effect of the OuDP can be increased by decreasing the distance between the hand and the lesion, increasing treatment duration, and increasing the treatment frequency [6,7].

Table 3. The efficacy of ipsilateral and contralateral hand to induce Ou MC decrescendo phenomenom (OuDP)

\begin{tabular}{|c|c|c|c|}
\hline \multirow{2}{*}{ Study } & Author (years) & \multicolumn{2}{|c|}{ Numbers of patients } \\
\cline { 3 - 4 } & & Ipsilateralhand & Contralateral hand \\
\hline$[4]$ & Ou et al. (2006-2009) & $3\left(0^{\mathrm{b}}\right)$ & $42\left(42^{\mathrm{b}}\right)$ \\
\hline$[6]$ & Ou et al. (2010-2011) & $39(0)$ & $39(36)$ \\
\hline$[6,7]$ & Ou et al. (2011-2014) & $3(0)$ & $40(40)$ \\
\hline Presenet study & Ou et al. (2015) & $11\left(1^{\mathrm{c}}\right)$ & $26(26)$ \\
\hline Total & & $56(1)$ & $147(144)$ \\
\hline
\end{tabular}

${ }^{\mathrm{a}}$ These 56 patients performed $\mathrm{Ou} \mathrm{MC}$ manipulation or handing remedy with ipsilateral and contralateral hand. Difference between contralteral and ipsilateral hand to induce OuDP, $P$ $<0.001$, Paired t test.

${ }^{\mathrm{b}}$ The number of patients with OuDP successfully induced.

cThe bilateral hip joint pain of one case was also relieved with ipsilateral hand 

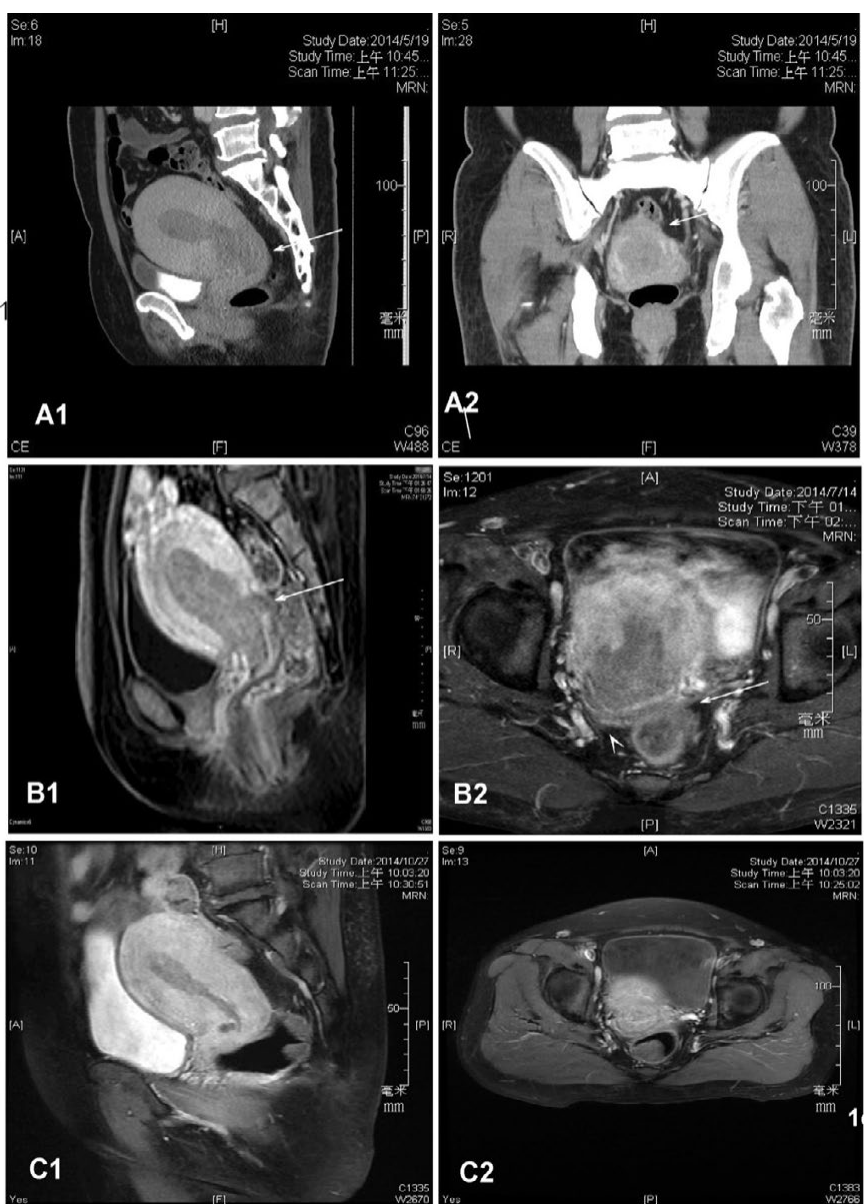

Figure 5. Female patient with uterine endometrioid carcinoma. A) Computed tomography (CT) on May 19, 2014. A1) Tumor involving the uterine cavity and cervix with suspected posterior uterine wall invasion (arrow) was noted. A2) Possible involvement of the posterior uterine wall (arrow). B) Magnetic resonance imaging (MRI) on July 14, 2014. B1) The lesion in the uterine cavity was approximately $8.9 \times 4.9 \times 4.5 \mathrm{~cm}$ (long axis $\times$ transverse $\times$ thickness), with posterior uterine wall invasion into the pelvis (arrow). B2) Uterine parametrial invasion (arrow head) and posterior wall involvement with loss of the fat layer between the uterine cervix and rectum (arrow). C) MRI on October 27, 2014 after application of the $\mathrm{Ou} \mathrm{MC}$ handing remedy. C1) No posterior wall involvement was noted, and the lesion was confined to the uterine cavity and measured approximately $6.7 \times 3.2 \times$ $1.5 \mathrm{~cm} . \mathrm{C} 2$ ) Uterine cervix with no evidence of a tumor.
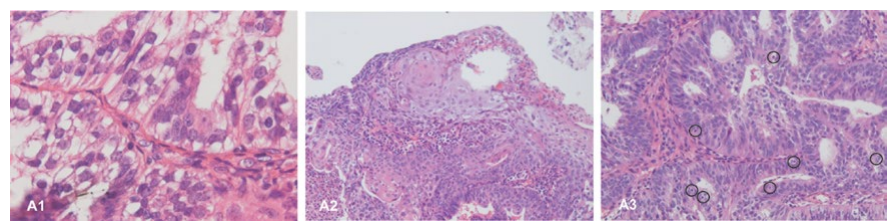

Figure 6. Endometrial biopsies of thepatient with uterine endometrioid carcinoma A1) Biopsy before treatment showed well-differentiated endometrioid carcinoma with secretory vacuoles with no definite squamous metaplasia or apoptosis-like lesions identified (hematoxylin and eosin [HE], magnification $\times 400$ ). A2, A3) Endometrial biopsy 29 days after treatment with the Ou MC handing remedy showed (A2) showed prominent squamous metaplasia of tumor cells and (A3) numerous apoptosis-like cells (black circles) (HE magnification $\times 100$ ).

The mechanism of action of Ou HR and OuDP remain unclear, but the potential roles of several sensory factors merit exploration because studies have shown inflammation proceeding bilaterally may occur through neurological transmission [2,3]. When the HR is performed, the treated area perceives the hand not only by proprioception or touch, but also by temperature changes. Elevation of tissue temperature by thermal therapy has been shown to increase cell-mediated cytotoxicity, which may have anti-bacterial, anti-parasitic, anti-viral, and antineoplastic effects when treating dermatological diseases [10]. However, 2 findings argue against the involvement of thermal effects in the OuDP. First, patient with cellulitis have responded to the HR despite not benefitting from far infrared ray therapy [6]. Second, when the ambient temperature during treatment is room temperature or below, the hand is usually cooler than the torso. The HR is effective when treating superficial lesions without contact with the body or lesion. Thus, touch may also not be essential to the effectiveness of the HR.

Spatial asymmetry, or establishment of cell polarity during embryogenesis by anteroposterior, dorsoventral, and left-right axes is crucial for normal tissue physiology and homeostasis. Loss of the polarity of cancer cells results in cells losing their ability to behave normally in response to physiological cues, and is frequently assumed to be a common feature of cancer progression. When the functions of tumor tissues are normalized, tumor cells may conform to regulation by apoptosis, growth suppression, and metastasis suppression, which normal cells undergo. The normalization of the tissue function of tumor
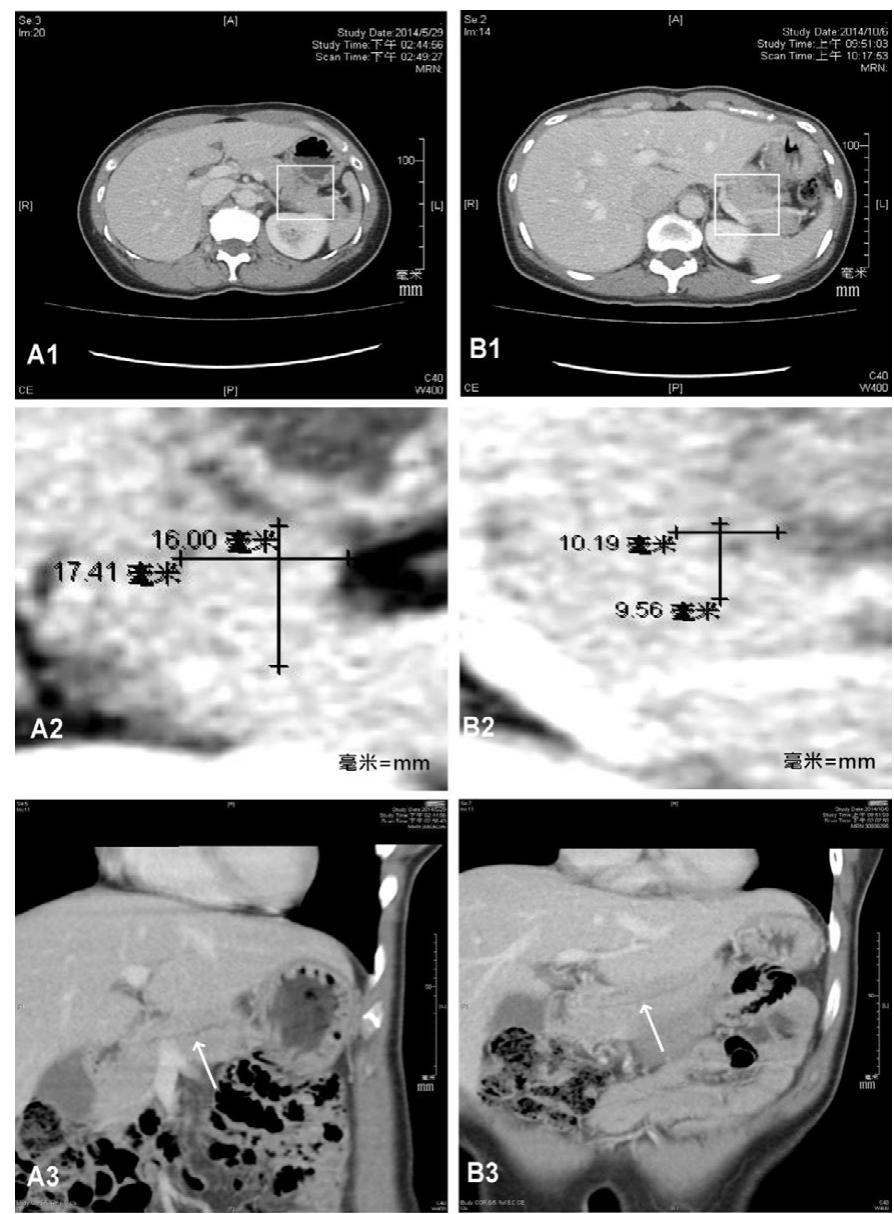

Figure 7. Computed tomography (CT) of patient with suspected pancreatic cancer. A) CT on May 29, 2014. A1) Anisodense nodule in the pancreatic tail was noted (square area) A2) Magnified image of the square area seen in A1: the size of the isodense nodule was approximately $1.6 \times 1.7 \mathrm{~cm}$. A3) Dilated tortuous main pancreatic duct with a diameter of approximately $0.39 \mathrm{~cm}$ (arrow). B) A CT scan on October 6, 2014 after treatment with the Ou MC handing remedy. B1) Theisodense nodule in pancreatic tail in the same position as the square area of A1. B2) Magnified image of the square area of B1 showed the size of the isodense nodule to be approximately $1.0 \times 1.0 \mathrm{~cm}$. B3) The main pancreatic duct of the pancreas was less tortuous with a diameter of approximately $0.14 \mathrm{~cm}$ (arrow). 
tissue may involve not only tumor cells, but also the microenvironment in which the tumor cells are located. The tumor microenvironment has been regarded as a main factor in tumor development and metastasis. The normalization of the tumor microenvironment subjects tumor cells to an environment that suppresses metastasis, prevents uninhibited proliferation, minimizes angiogenesis, and eliminates abnormal cells via the normalized host immunological system [11,12].

Embryological development of animals usually patterns along the embryonic axes with regard to the functional features developed during early embryonic life. Recent studies have shown the signaling system of embryonic axes imparts polarization of individual cells leading to normal function [13]. Most human cells and tissue in adults are polarized to have normal functions. Linguistic studies have demonstrated that a space-time congruency effect exists from the left to right that is coordinated with the left-right axis of the human body [14]. This indicates there are mutual interactions of the left to right axis between individuals that may result in a physiological response. As previously mentioned, animal study has that claw changes can be caused by mutual conspecific interactions among snapping shrimps [1]. The Ou HR is effective at producing the OuDP with the contralateral hand, but not the ipsilateral hand. This finding implies that the axes of embryonic polarity are the potential mechanism underlying the OuDP.

Although the occurrence of human cancer, including breast, lung, kidney, testis, and melanoma have been shown to have left-right asymmetry, the mechanism underlying this phenomenon remains unexplained in modern medicine [15]. However, this may suggest that this disease may be caused by abnormal fetal axes development, which can be corrected by the OuDP. Most human cells and tissues in adults have been found to be polarized such that normal function can be achieved [16]. Pituitary homeobox 2 (PITX2), NODAL, and LEFTY-1 are important genes controlling embryonic development along the left-right axis in mammals, and have been found to be related to the development or suppression of various cancers, suggesting they may have therapeutic relevance [17-19]. For example, PITX2 protects renal cancer cell lines against doxorubicin toxicity, NODAL promotes breast cancer growth or progression, and suppression of LEFTY-1 expression induces pluripotent cancer cells [17-19]. A large number of signaling molecules that are active during the specification of embryonic leftright asymmetry are also active during tumor progression. It can be hypothesized that cancer lateralization is associated with signaling molecules relating to embryonic left-right axis development. Cancer regression induced by the OuDP using the contralateral hand may also indicate the importance of embryonic polarity to cancer development. The anti-inflammatory effect of the OuDP may also facilitate the regression of cancer [20], but contemporary anti-inflammatory treatments for cancer only show the capability to prevent cancer or minimize cancer-associated inflammatory symptoms [21,22].

While the Ou HR is effective for treating a wide variety of diseases, there are still many unresolved questions. Our prior studies showed that the OuDP not always be effectively induced by the examiner, and alone it may not always be sufficient to resolve diseases [6]. The HR may cause regression of malignant changes for certain cancers, but it has not been evaluated with benign tumors. While the OuDP produces a restorative effect on the opposite side of the body, additional studies are required to determine whether it may excessively enhance host responses and produce any harmful effects [3]. The effect of the Ou HR for open wounds and critically ill patients has not been evaluated, and the Ou HR may contaminate an open wound. In addition, the appropriate duration and frequency of HR application for the treatment

\section{Interaction of human bilateral parts}

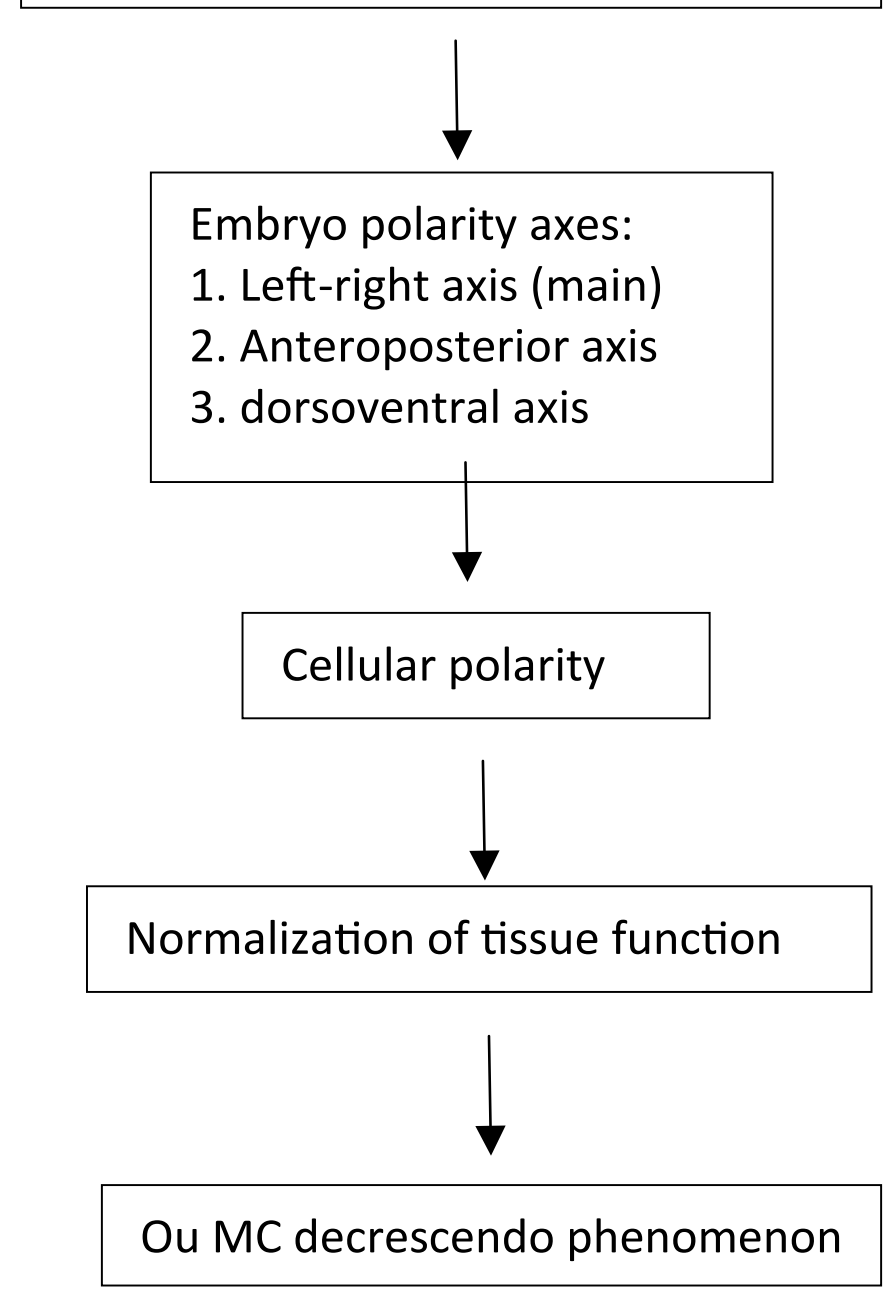

Figure 8. Possible role of embryonic polarity axes for the normalization of tissue function induced by the interaction between human bilateral parts.

of different diseases requires further study, as does whether emotional disturbances affect the efficacy of the technique [6].

The primary limitations of this report are the small number of cases, and that patients were not randomized to receive the HR or conventional care. Patients chose to receive the HR, and this naturally imparts bias into a case series. It should be noted that in the current series as well as in the prior reports application of the HR with the ipsilateral hand produced no prominent effect, whereas use of the contralateral hand in the same patients was effective. However, OuDP is probably induced by anteroposterior, dorsoventral, and left-right axes, and ipsilateral may not be always ineffective.

\section{Conclusion}

The Ou HR and OuDP produce a reproducible effect to accelerate the recovery of infections, inflammation, degenerative diseases, organ dysfunction, and malignant changes [23-25]. The effect mainly occurs with the use of the contralateral hand-use of the ipsilateral hand produces no prominent effect in most occasions, and can be induced by the patient themselves or by a therapist. Studies have shown the 
signaling system in the development of embryonic axes may impart polarization of the behaviors of individual cells in adults, which leads to normalization of cell function. The OuDP induced via the interaction between human bilateral parts also results in normalization of tissue function suggesting that the axes of embryonic polarity may impart the OuDP (Figure 8).

\section{Acknowledgements}

The authors thank the encouraging and spiritual support from Ms. Ou Yi-Jen.

\section{References}

1. Pearce J, Govind CK (1987) Spontaneous generation of bilateral symmetry in the paired claws and closer muscles of adult snapping shrimps. Development 100: 57-63.

2. Murray FS, Weaver MM (1975) Effects of ipsilateral and contralateral counterirritation on experimentally produced itch in human beings. J Comp Physiol Psychol 89: 819826. [Crossref]

3. Shenker N, Haigh R, Roberts E, Mapp P, Harris N, et al. (2003) A review of contralateral responses to a unilateral inflammatory lesion. Rheumatology (Oxford) 42: 1279-1286. [Crossref]

4. Ou MC, Pang CC, Ou D, Su CH (2012) The implications of abdominal palpation with Ou MC manipulation for women with acute abdomen. Am J Emerg Med 30: 421-425. [Crossref]

5. Ou MC, Pang CC, Ou D (2011) Abdominal palpation with Ou MC manipulation (APOM) for women with acute abdomen caused by pelvic inflammatory disease: a pilot study. J Emerg Med 41: 87-89. [Crossref]

6. Ou MC, Ou D, Pang CC (2014) A primitive approach to Ou MC decrescendo phenomenon with a hands-on therapy-The relation between human bilateral symmetry and disease. Natural Science 6: 88-98.

7. Ou M C, Ou D, Pang CC (2015) The preliminary approach for the human cancer regression by the interaction between human bilateral symmetrical parts - Ou MC decrescendo phenomenon. Natural Science 7: 71-78.

8. Department of Health, Executive Yuan, Taiwan ROC (1993) No. 82075656, 1993. [Available at: http://homepagevghtpegovtw/ ged/listn/listn_a122htm.]

9. Yao X, Li H, Leng SX (2011) Inflammation and immune system alterations in frailty. Clin Geriatr Med 27: 79-87. [Crossref]

10. Badgwell Doherty C, Doherty SD, Rosen T (2010) Thermotherapy in dermatologic infections. J Am Acad Dermatol 62: 909-927. [Crossref]
11. Lorusso G, Rüegg C (2008) Thetumor microenvironment and its contribution to tumor evolution toward metastasis. Histochem Cell Biol 130: 1091-1103. [Crossref]

12. Dunn GP, Koebel CM, Schreiber RD (2006) Interferons, immunity and cancer immunoediting. Nat Rev Immunol 6: 836-848. [Crossref]

13. Gray RS, Roszko I, Solnica-Krezel L (2011) Planar cell polarity: coordinating morphogenetic cell behaviors with embryonic polarity. Dev Cell 21: 120-133. [Crossref]

14. Torralbo A, Santiago J, Lupiáñez J (2006) Flexible conceptual projection of time onto spatial frames of reference. Cogn Sci 30: 745-757. [Crossref]

15. Wilting J, Hagedorn M (2011) Left-right asymmetry in embryonic development and breast cancer: common molecular determinants? Curr Med Chem 18: 5519-5527. [Crossref]

16. D'Angelo A, Franco B (2009) The dynamic cilium in human diseases. Pathogenetics 2: 3. [Crossref]

17. Lee WK, Chakraborty PK, Thévenod F (2013) Pituitary homeobox 2 (PITX2) protects renal cancer cell lines against doxorubicin toxicity by transcriptional activation of the multidrug transporter ABCB1. IntJ Cancer 133: 556-567. [Crossref]

18. Quail DF, Zhang G, Walsh LA, Siegers GM, Dieters-Castator DZ, et al. (2012) Embryonic morphogen nodal promotes breast cancer growth and progression. PLoS One 7: e48237. [Crossref]

19. Saito A, Ochiai H, Okada S, Miyata N, Azuma T (2013) Suppression of Lefty expression in induced pluripotent cancer cells. FASEB J 27: 2165-2174. [Crossref]

20. Aggarwal BB, Shishodia S, Sandur SK, Pandey MK, Sethi G (2006) Inflammation and cancer: how hot is the link? Biochem Pharmacol 72: 1605-1621. [Crossref]

21. Roxburgh CS, McMillan DC (2014) Cancer and systemic inflammation: treat the tumour and treat the host. Br J Cancer 110: 1409-1412. [Crossref]

22. Shebl FM, Hsing AW, Park Y, Hollenbeck AR, Chu LW, et al. (2014) Non-steroida anti-inflammatory drugs use is associated with reduced risk of inflammation-associated cancers: NIH-AARP study. PLoS One 9: e114633. [Crossref]

23. Ou, MC, Pang CC, Ou D (2012) A primitive approach for Ou MC handing remedy with a comparison to the ancient laying on of hands. In: Abstract book of the $15^{\text {th }}$ Annual Meeting of the British Infection Association: 9. London.

24. Ou MC, Ou D, Pang CC (2014) The relation between human bilateral symmetry and disease. Proceedings of the Physiological Society 31: PCA132.

25. Ou MC, Ou D, Pang CC (2015) The potential mechanism of bilateral human symmetric parts interaction for the degenerative and oncological diseases. Proceedings of the Physiological Society 33: PC08.

Copyright: (C) 2015 Ou MC. This is an open-access article distributed under the terms of the Creative Commons Attribution License, which permits unrestricted use, distribution, and reproduction in any medium, provided the original author and source are credited. 